PDFlib PLOP: PDF Linearization, Optimization, Privacy

Page inserted by evaluation version www.pdflib.com - sales@pdflib.com 


\title{
Structural, Magnetic, and Theoretical Characterization of a Heterometallic Polypyridylamide Complex**
}

\author{
Marie-Madeleine Rohmer,* Isiah Po-Chun Liu, Jheng-Cheng Lin, Mei-Jyun Chiu, Chia- \\ Hwa Lee, Gene-Hsiang Lee, Marc Bénard, Xavier López, and Shie-Ming Peng*
}

One-dimensional complexes with metal-containing backbones have been attractive to physicists and chemists for several decades, ${ }^{[1]}$ and their interest has been further increased in the last ten years by potential applications in nanoelectronics. ${ }^{[2]}$ Apart from the family of platinum and iridium blues, most chains of metal atoms were constrained to be aligned by means of purposely designed ligands. ${ }^{[3,4]}$ The polypyridylamido ligands have produced to date the widest variety of metal chains with controlled lengths, as far as the nature and the number of metal atoms are concerned. ${ }^{[3]}$ Strings with three to nine metal atoms were obtained with $\mathrm{Cr}$ and Ni. Chains containing three or five metal atoms, characterized for $\mathrm{Co}, \mathrm{Cu}, \mathrm{Ru}$, and $\mathrm{Rh}$, have unprecedented structural versatility and magnetic behavior that is hard to predict. ${ }^{[3]}$ Even though heterometallic chain complexes are not uncommon ${ }^{[5]}$ and often exhibit similarity to platinum blues $^{[6]}$ or involve weak noncovalent interactions between heavy atoms, ${ }^{[7]}$ all linear complexes with polypyridylamido or closely related ligands characterized to date incorporate a single type of metal atoms. We report here the synthesis, $\mathrm{X}$ ray structure, and magnetic properties of $\left[\mathrm{Co}_{2} \mathrm{PdCl}_{2}(\mathrm{dpa})_{4}\right](\mathbf{1}$; $\mathrm{dpa}=$ bis(2-pyridyl)amide), the first trinuclear complex of

[*] Dr. M.-M. Rohmer, Dr. M. Bénard, Dr. X. López ${ }^{[+]}$

Laboratoire de Chimie Quantique, Institut de Chimie

UMR 7177 CNRS - Université Louis Pasteur

4 rue Blaise Pascal, 67070 Strasbourg Cedex (France)

Fax: (+33) 3-9024-1589

E-mail:mmrohmer@quantix.u-strasbg.fr

I. P.-C. Liu, Dr. J.-C. Lin, M.-J. Chiu, C.-H. Lee, G.-H. Lee,

Prof. Dr. S.-M. Peng

Department of Chemistry, National Taiwan University

Taipei, 106, Taiwan (ROC)

Fax: (+886) 2-8369-3765

E-mail: smpeng@ntu.edu.tw

I. P.-C. Liu, Prof. Dr. S.-M. Peng

Institute of Chemistry, Academia Sinica

Taipei, 115, Taiwan (ROC)

$\left.{ }^{+}\right]$Current address:

Depart. de Quimica Fisica i Inorganica

Universitat Rovira i Virgili, Campus Sescelades

c. Marcel.li Domingo s/n, Tarragona 43007 (Spain)

[***] This work was supported by the CNRS (France) and the NRC (Taiwan) through the joint Research Project no. 16189 "Modelling Nanowires". Calculations were carried out at the IDRIS center (Orsay, France). We thank Prof. Hiroshi Sakiyama (Yamagata University, Japan) for valuable discussions about magnetism. X.L. thanks the Government of Spain for a postdoctoral fellowship (no. EX-2004-0113).

$\square$ Supporting information for this article is available on the WWW under http://www.angewandte.org or from the author. dpa with a heteronuclear backbone. The magnetic behavior of 1 was investigated and found to be consistent with a complex interaction involving zero-field splitting superimposed on strong exchange coupling between two high-spin $\mathrm{Co}^{\mathrm{II}}$ ions separated by a diamagnetic $\mathrm{Pd}$ center. The ground-state electronic structure of $\mathbf{1}$ was interpreted by means of DFT calculations.

Complex 1 was synthesized by a variant of the protocol currently used to obtain trinuclear dipyridylamide complexes. ${ }^{[8]}$ Single-crystal X-ray analysis of $\mathbf{1} \cdot\left(\mathrm{C}_{2} \mathrm{H}_{5}\right)_{2} \mathrm{O}$ resulted in the structure displayed in Figure 1 . The heterometallic

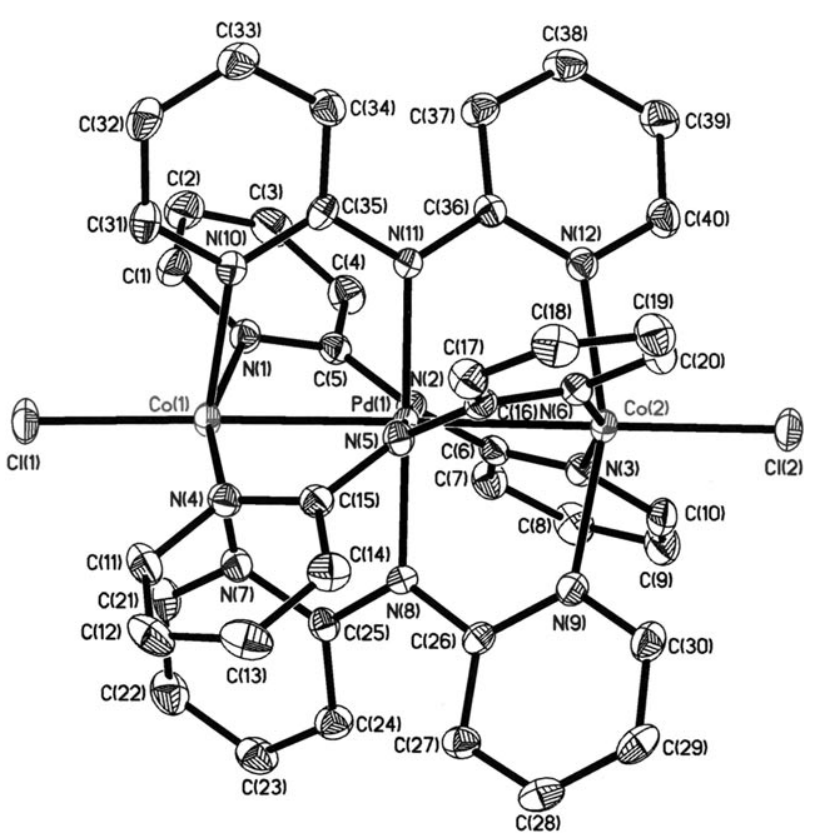

Figure 1. ORTEP view of the molecular structure of 1 (50\% probabil-

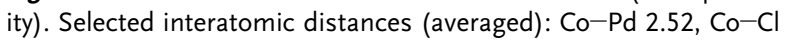
2.34, Co-N 2.16, Pd-N $2.01 \AA$.

chain is helically wrapped by four syn-syn-type ligands, and the complex exhibits approximate $D_{4}$ symmetry. The average $\mathrm{Co}-\mathrm{N}(2.16 \AA)$ and $\mathrm{Co}-\mathrm{Cl}(2.34 \AA)$ distances are quite similar to those observed at room temperature for the isolated, highspin $\mathrm{Co}^{\mathrm{II}}$ ion in the unsymmetrical form of $\left[\mathrm{Co}_{3} \mathrm{Cl}_{2}(\mathrm{dpa})_{4}\right] .{ }^{[9]}$ The average $\mathrm{Pd}-\mathrm{N}(2.01 \AA)$ is much shorter than the average $\mathrm{Co}-\mathrm{N}$ distance and suggests that the $\mathrm{Pd}$ atom is essentially low-spin $(S=0)$ in square-planar coordination. The relatively short Pd-Co distance $(2.52 \AA)$ is consistent with a weak metal-metal interaction, as confirmed by DFT calculations. 
The temperature-dependent magnetic susceptibility of $\mathbf{1}$ was investigated (Figure 2). With decreasing temperature, the $\chi_{\mathrm{M}} T$ value decreases rapidly in an unusual linear fashion. This behavior can be ascribed to an antiferromagnetic interaction

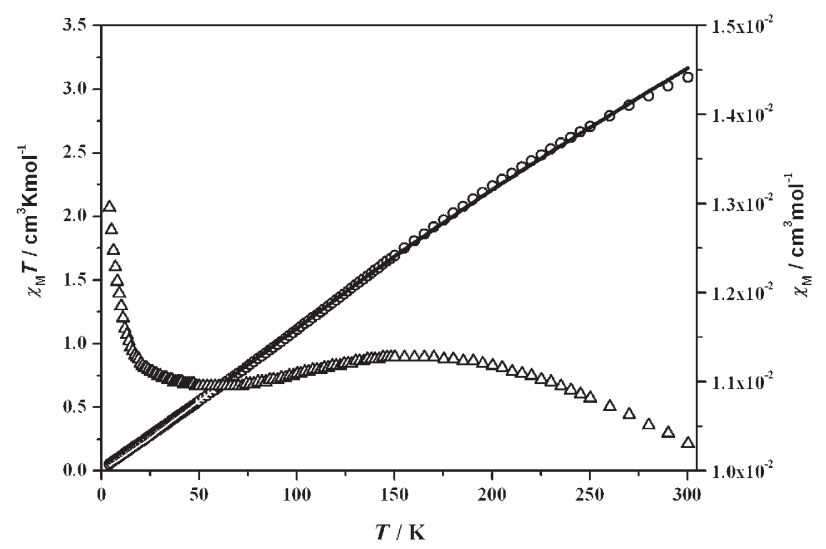

Figure 2. Temperature dependence of $\chi_{M} T(\circ)$ and $\chi_{M}(\triangle)$ of 1 . The black line shows the best fit of $\chi_{M} T$ (see text). The $\chi_{M}$ data below $25 \mathrm{~K}$ are probably affected by a paramagnetic impurity.

modified by the contribution of anisotropic exchange. The $\chi_{\mathrm{M}} T$ value at $300 \mathrm{~K}\left(3.09 \mathrm{~cm}^{3} \mathrm{~K} \mathrm{~mol}^{-1}\right)$ is smaller than expected for two isolated $S=3 / 2$ spins $\left(3.75 \mathrm{~cm}^{3} \mathrm{~K} \mathrm{~mol}^{-1}\right)$, and this can be interpreted as resulting from significant antiferromagnetic coupling. A satisfactory fit (Figure 2) was obtained with a model including the isotropic Heisenberg Hamiltonian plus an axial zero-field splitting operator [Eq. (1)]. ${ }^{[10]}$

$\hat{H}=-2 J \hat{S}_{1} \hat{S}_{2}+D\left[\hat{S}_{z}^{2}-1 / 3 S(S+1)\right]$

The best-fit parameters are $g=2.20,2 J=-92.47 \mathrm{~cm}^{-1}$, $|D|=50.95 \mathrm{~cm}^{-1}, \chi_{\mathrm{TIP}}=0.01 \mathrm{~cm}^{3} \mathrm{~mol}^{-1}$, and $R^{2}=0.99$. The temperature-independent paramagnetism (TIP) was included in this fitting procesure to describe the coupling between ground and ecited states. The value obtained for $|D|$ is similar to those derived for various five- or six-coordinate high-spin mono- and dicobalt(II) complexes. ${ }^{[10]}$ The data measured for $\chi_{\mathrm{M}}$ below $25 \mathrm{~K}$ varied significantly with the sample and gave rise to a sharp increase in $\chi_{M}$ that should be assigned to the presence of a paramagnetic impurity in variable amounts.

The DFT/B3LYP calculations were carried out on 1 with the Gaussian 03 software package. ${ }^{[11]}$ Previous calculations on the symmetric $(s)$ form of $\left[\mathrm{Co}_{3} \mathrm{Cl}_{2}(\mathrm{dpa})_{4}\right]$ and other $\left[\mathrm{M}_{3} \mathrm{Cl}_{2}-\right.$ $\left.(\mathrm{dpa})_{4}\right]$ complexes $^{[12]}$ have clarified the shape and the sequence of the metal orbitals in these complexes under the assumption of $D_{4}$ symmetry (Figure 3 ).

In the molecular ground state, the occupancies of the highest MOs depend on the competition between the scheme of a metal chain exhibiting a strong, delocalized interaction, and that of an assembly of weakly interacting metal atoms. Various electronic configurations referring to both models were computed for $\mathbf{1}$, and their geometries optimized. For
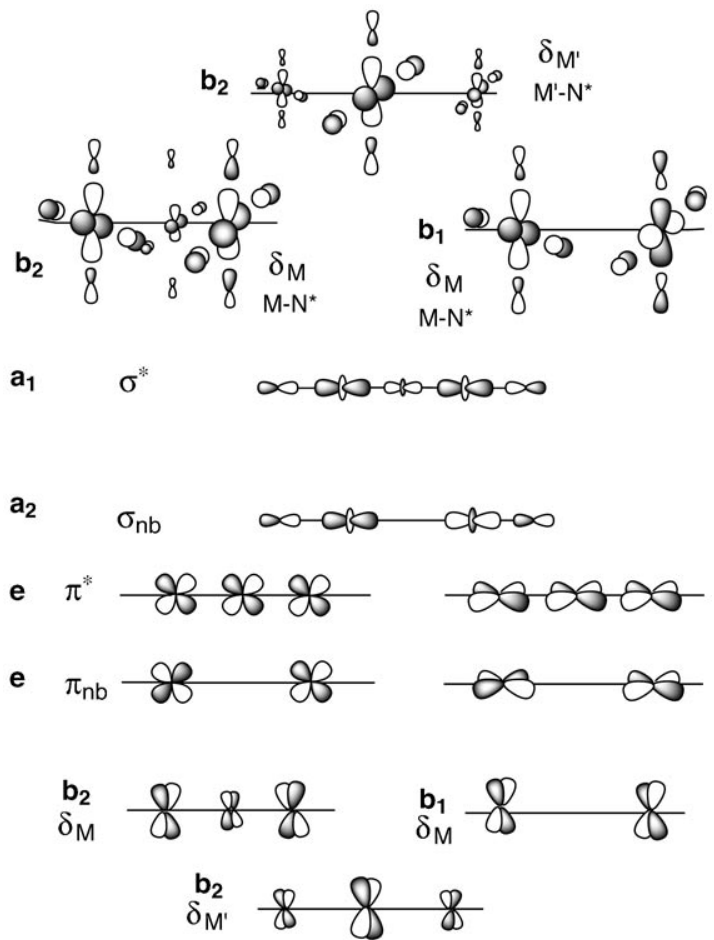

- $\pi$ \& \& \& $-\infty \infty$

$a_{1}$

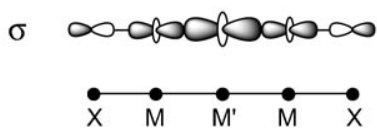

Figure 3. Sequence of metal orbitals in the closed-shell electronic configurations of $s-\left[\mathrm{Co}_{3} \mathrm{Cl}_{2}(\mathrm{dpa})_{4}\right]$ and $\left[\mathrm{Co}_{2} \mathrm{PdCl}_{2}(\mathrm{dpa})_{4}\right]\left(D_{4}\right.$ symmetry).

each configuration, Figure 4 displays the distribution of the metal electrons among the 15 metal valence orbitals, together with the relative energy of each configuration. The main geometrical parameters associated with each configuration are given in the Supporting Information.

In agreement with the latter model, the magnetic behavior observed for 1 suggests that the ground state results from antiferromagnetic coupling between two high-spin $(S=3 / 2)$ $\mathrm{Co}^{\mathrm{II}}$ ions, separated by a magnetically inactive $\mathrm{Pd}^{\mathrm{II}}$ center. The resulting molecular state of maximal spin is therefore a septet $\left({ }^{7} \mathrm{~A}_{2}\right)$ with six unpaired electrons largely localized on the terminal metal atoms. This state was indeed found to be lowest in energy in symmetry-adapted calculations, that is, without considering any magnetic coupling between unpaired electrons (Figure 4). The geometry optimized for the ${ }^{7} \mathrm{~A}_{2}$ state agrees well with the observed structure. For the two other electronic states displayed in Figure 4, the optimal distances are strongly at variance with experiment (Supporting Information).

As long as the electronic ground state of the magnetic centers is nondegenerate, coupling between spins $S_{1}$ and $S_{2}$ of two transition metals is appropriately modeled by means of the isotropic Heisenberg Hamiltonian $\hat{H}=-2 J_{12} \hat{S}_{1} \cdot \hat{S}_{2}$. The case of high-spin $\mathrm{Co}^{\mathrm{II}}\left({ }^{4} \mathrm{~T}_{1 \mathrm{~g}}\right.$ state $)$ is more complicated, 


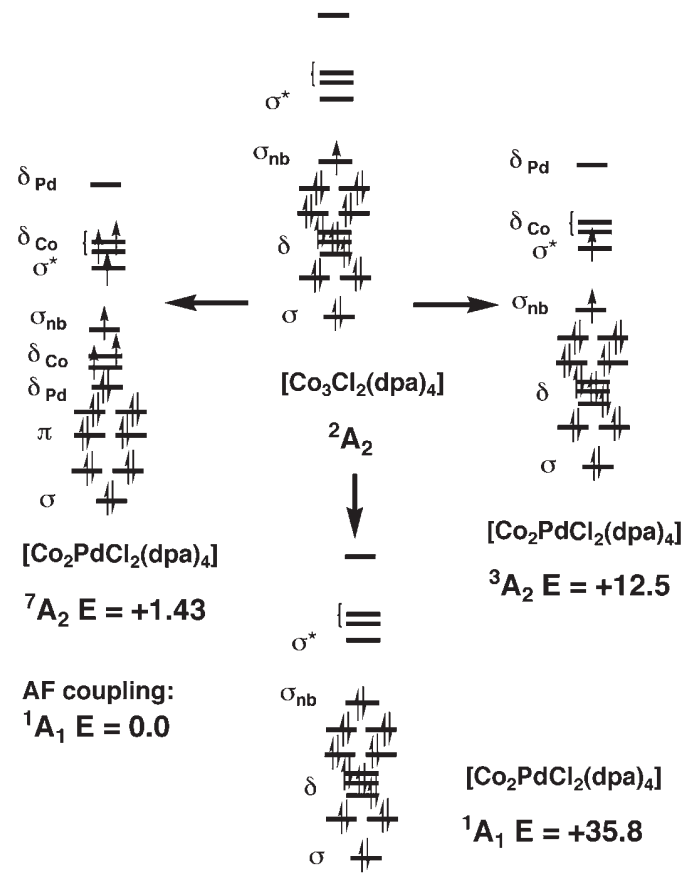

Figure 4. Lowest electronic configuration for $s-\left[\mathrm{Co}_{3} \mathrm{Cl}_{2}(\mathrm{dpa})_{4}\right]$ and three competing electronic configurations for 1 . Relative energies [ $\mathrm{kcal} \mathrm{mol}^{-1}$ ] are given with respect to the broken-symmetry state resulting from antiferromagnetic (AF) coupling of six unpaired electrons.

however, due to the combination of orbital and spin degeneracies. A convenient treatment requires the introduction of spin-orbit coupling terms in the expression of the Hamiltonian, which then becomes symmetry-dependent. ${ }^{[10,13]}$ Most recent treatments of magnetic susceptibility rely on the assumption that the isotropic exchange coupling is small compared to the energy gap between the lowest energy levels generated from the ${ }^{4} \mathrm{~T}_{1 \mathrm{~g}}$ atomic ground state by spin-orbit coupling. ${ }^{[14-16]}$ To our knowledge, all dinuclear $\mathrm{Co}^{\mathrm{II}}$ complexes with atomic spins $3 / 2$ investigated to date satisfy the latter condition, with $-2 J$ values of less than $20 \mathrm{~cm}^{-1}$. ${ }^{[13,14,16]}$

The development of a complete theoretical analysis of the magnetism in $\mathbf{1}$ is far beyond the scope of this communication. However, within the framework of the present DFT/B3LYP calculations, we can provide a rough estimate of the exchange coupling parameter, assumed to be isotropic. The method used for this calculation is the broken-symmetry (BS) approach of Noodleman et al. ${ }^{[17]}$ based on the classical Heisenberg Hamiltonian. This method does not account for the orbitally dependent corrections to the exchange interactions. $^{[14]}$

The unpaired electrons localized on the Co atoms in the ${ }^{7} \mathrm{~A}_{2}$ configuration can be coupled into an antiferromagnetic singlet ground state. The spin-polarized singlet state derived from this coupling was further stabilized by $1.43 \mathrm{kcal} \mathrm{mol}^{-1}$ with respect to the ${ }^{7} \mathrm{~A}_{2}$ configuration. After projection onto the proper spin eigenstate, ${ }^{[18]}$ the computed value of $-2 J$ is $113.3 \mathrm{~cm}^{-1}$, far beyond the values currently encountered for the exchange coupling parameter in dinuclear complexes of $\mathrm{Co}^{\mathrm{II}}$. The order of magnitude of the exchange coupling then becomes comparable with the spin-orbit coupling constant $\lambda$ $\left(\lambda=-172 \mathrm{~cm}^{-1}\right.$ for the free $\mathrm{Co}^{\mathrm{II}}$ ion).

Another set of low-energy states implies a low-spin $(S=$ $1 / 2$ ) configuration for $\mathrm{Co}^{\mathrm{II}}$. The molecular state of maximal spin is then a triplet $\left({ }^{3} \mathrm{~A}_{2}\right)$ in which the unpaired electrons are accommodated in the two highest $\sigma$-type MOs (Figure 4). This state was computed to be $12.5 \mathrm{kcal} \mathrm{mol}^{-1}$ above the ground state, and coupling between the two unpaired electrons yields a second antiferromagnetic singlet, at $+9.5 \mathrm{kcal} \mathrm{mol}^{-1}$.

Finally, we considered an electronic state characterized by delocalized bonding interaction along the metal framework. Such a state is deduced from the doublet ground state of $s$ $\left[\mathrm{Co}_{3} \mathrm{Cl}_{2}(\mathrm{dpa})_{4}\right]$ by accommodating the extra electron of $\mathrm{Pd}^{\mathrm{II}}$ on the nonbonding (nb) $\sigma \mathrm{MO}$ (Figure 4). This yields a closedshell state with $\sigma_{\mathrm{nb}}$ as the HOMO that displays a fourelectron/three-center bond. Surprisingly, this state lies as much as $36 \mathrm{kcal} \mathrm{mol}^{-1}$ above the ground state (Figure 4). The reason for this destabilization should be looked for in the unbalanced $\mathrm{Co} \leftarrow \mathrm{Pd} \rightarrow$ Co charge transfer implied by the lack of contribution from $\mathrm{Pd}$ to the $\sigma_{\mathrm{nb}} \mathrm{MO}$ (Figure 3). The diamagnetic state therefore entails a formal oxidation state of III for Pd, with concomitant reduction of the neighboring Co atoms. Such an unfavorable reorganization of the atomic electron density obviously offsets the benefit of delocalized bonding.

In conclusion, we have prepared and characterized an unprecedented metal-chain complex with heterometallic $\mathrm{Co} /$ Pd core. Both the crystal structure and the magnetic measurements suggest that the ground state results from coupling between the $\mathrm{Co}^{\mathrm{II}}$ ions, both in high-spin configuration. Density functional calculations confirm this assignment and predict a relatively large isotropic exchange interaction. However, the orbital degeneracy characteristic of high-spin $\mathrm{Co}^{\mathrm{II}}$ requires that spin-orbit coupling terms and anisotropic corrections are taken into account. The electronic ground state of $\left[\mathrm{Co}_{2} \mathrm{PdCl}_{2}(\mathrm{dpa})_{4}\right]$ and the magnetic behavior of $\mathrm{Co}^{\mathrm{II}}$ in this complex are therefore strongly at variance with those observed in the tricobalt homologue. This suggests that the accommodation of the additional electron provided by the central palladium ion in the $\sigma$ nonbonding $\mathrm{MO}$ centered on the terminal cobalt ions offsets the advantage of a delocalized bond along the metal chain. The insertion of one or several heterocenter(s) in a string of metal atoms of definite length could therefore provide a new way of tuning its magnetic and conducting properties.

\section{Experimental Section}

Synthesis: Full experimental details and analytical data can be found in the Supporting Information

Crystal data for 1: $\mathrm{Co}_{2} \mathrm{PdC}_{44} \mathrm{H}_{42} \mathrm{~N}_{12} \mathrm{Cl}_{2} \mathrm{O}, M=1050.06$, monoclinic space group $P 2_{1} / c, a=16.0656(9), b=15.7468(8), c=17.0694(9) \AA$, $\alpha=90, \quad \beta=97.895(1), \quad \gamma=90^{\circ}, \quad V=4277.3(4) \AA^{3}, \quad Z=4, \quad \rho_{\text {calcd }}=$ $1.631 \mathrm{mg} \mathrm{m}^{-3}, R_{1}=0.0297, w R_{2}=0.0688$. Details on data collection are given in the Supporting Information. CCDC 623473 contains the supplementary crystallographic data for this paper. These data can be obtained free of charge from The Cambridge Crystallographic Data Centre via www.ccdc.cam.ac.uk/data_request/cif. 
DFT/B3LYP calculations: Details of the used atomic basis sets can be found in the Supporting Information.

Received: October 20, 2006

Revised: November 22, 2006

Published online: March 27, 2007

Keywords: density functional calculations - heterometallic complexes - magnetic properties - metal-metal interactions · $\mathrm{N}$ ligands

[1] Extended Linear Chain Compounds, Vols. 1-3 (Ed.: J. S. Miller) Plenum, New-York, 1982.

[2] a) D. H. Chae, J. F. Berry, F. A. Cotton, C. A. Murillo, Z. Yao, Nano Lett. 2006, 6, 165; b) I.-W. P. Chen, M.-D. Fu, W.-H. Tseng, J.-Y. Yu, S.-H. Wu, C.-J. Ku, C.-h. Chen, S. M. Peng, Angew. Chem. 2006, 118, 5946; Angew. Chem. Int. Ed. 2006, 45, 5814; Erratum: I.-W. P. Chen, M.-D. Fu, W.-H. Tseng, J.-Y. Yu, S.-H. Wu, C.-J. Ku, C.-h. Chen, S. M. Peng, Angew. Chem. 2006, 118, 6390; Angew. Chem. Int. Ed. 2006, 45, 6244.

[3] J. F. Berry in Multiple Bonds between Metal Atoms, 3rd ed. (Eds.: F. A. Cotton, C. A. Murillo, R. A. Walton), Springer Science, New York, 2005

[4] J. K. Bera, K. R. Dunbar, Angew. Chem. 2002, 114, 4633; Angew. Chem. Int. Ed. 2002, 41, 4453.

[5] a) W. Zhang, A. Behrens, J. Gätjens, M. Ebel, X. Wu, D. Rehder, Inorg. Chem. 2004, 43, 3020; b) Z.-X. Zhang, Y. Li, K.-C. Li, W.D. Song, Q.-S. Li, H.-P. Xiong, J.-Q. Xu, L.-Y. Pan, J. Mol. Struct. 2006, 791, 117; c) N. Amir, M. Motonishi, M. Fujita, Y.
Miyashita, K. Fujisawa, K. Okamoto, Eur. J. Inorg. Chem. 2006, 1041.

[6] T. Tanase, R. A. Begum, Organometallics 2001, 20, 106.

[7] a) L. R. Falvello, J. Forniés, R. Garde, A. García, E. Lalinde, M. T. Moreno, A. Steiner, M. Tomás, I. Usón, Inorg. Chem. 2006, 45, 2543; b) E. J. Fernández, A. Laguna, J. M. López-de-Luzuriaga, M. Montiel, M. E. Olmos, J. Pérez, Organometallics 2006, $25,1689$.

[8] a) E.-C. Yang, M.-C. Cheng, M.-S. Tsai, S.-M. Peng, J. Chem. Soc. Chem. Commun. 1994, 2377; b) F. A. Cotton, L. M. Daniels, C. A. Murillo, I. Pascual, J. Am. Chem. Soc. 1997, 119, 10223.

[9] R. Clérac, F. A. Cotton, L. M. Daniels, K. R. Dunbar, K. Kirschbaum, C. A. Murillo, A. A. Pinkerton, A. J. Schultz, X. Wang, J. Am. Chem. Soc. 2000, 122, 6226.

[10] a) O. Kahn, Molecular Magnetism, VCH, New York, 1993; b) R. Boča, Coord. Chem. Rev. 2004, 248, 757.

[11] Gaussian 03 (Revision B.05): M. J. Frisch et al., see Supporting Information

[12] P. Kiehl, M.-M. Rohmer, M. Bénard, Inorg. Chem. 2004, 43, 3151.

[13] K. Fink, C. Wang, V. Staemmler, Inorg. Chem. 1999, 38, 3847.

[14] S. M. Ostrovsky, K. Falk, J. Pelikan, D. A. Brown, Z. Tomkowicz, W. Haase, Inorg. Chem. 2006, 45, 688.

[15] H. Sakiyama, Inorg. Chim. Acta 2006, 359, 2097.

[16] a) M. J. Hossain, M. Yamasaki, M. Mikuriya, A. Kuribayashi, H. Sakiyama, Inorg. Chem. 2002, 41, 4058; b) H. Sakiyama, J. Chem. Software 2001, 7, 171.

[17] L. Noodleman, C. Y. Peng, D. A. Case, J. M. Mouesca, Coord. Chem. Rev. 1995, 144, 199.

[18] M. Mitani, H. Mori, Y. Takano, D. Yamaki, Y. Yoshioka, K. Yamaguchi, J. Chem. Phys. 2000, 113, 4035. 